\title{
Concaving AgI Sub-Microparticles for Enhanced Photocatalysis
}

Changhua Ana, ${ }^{\text {a, Junxue Liu }}{ }^{\mathrm{a}}$, Shutao Wanga, Jun Zhang ${ }^{\mathrm{a}}$, Zhaojie Wanga ${ }^{\mathrm{a}}$, Ran Long ${ }^{\mathrm{c}}$, and Yugang Sun $^{\mathrm{b}, *}$


Science, China University of Petroleum, Qingdao, 266580, P. R. China

Fax: (+86) 532-8698-1787, E-mail: anchh@upc.edu.cn (Changhua An)

${ }^{\mathrm{b}}$ Center for Nanoscale Materials, Argonne National Laboratory, 9700 South Cass Avenue, Argonne, Illinois 60439, USA

Fax: (+1) 630-252-4646 E-mail: ygsun@anl.gov (Yugnag Sun)

${ }^{c}$ School of Chemistry and Materials Science, University of Science and Technology of China, Hefei, Anhui 230026, P. R. China 


\begin{abstract}
Concave particles represent a new class of structures with their surfaces curving in or hollowed inward and thus presence of regions with negative curvatures. Owing to the potential high-index facets and negative curvatures, crystalline particles with concave surfaces are expected to show unexplored or substantially enhanced performance in comparison with the counterparts with convex surfaces. In this report, we highlight a facile approach for the first-time synthesis of concave AgI nanoparticles through a controlled etching of spherical AgI particles in a solution containing ethylenediamine, absolute alcohol, and polyvinylpyrrolidone. Physical parameters including morphology and size of the resulting concave AgI particles can be tuned by carefully controlling the reaction conditions such as the amount of precursors and the injection rate of precursor solutions. Most importantly, the concave AgI particles exhibit a much higher efficiency towards photocatalytic degradation of organic molecules than the corresponding spherical AgI particles. The as-synthesized concave AgI particles are expected to be useful not only for the fundamental investigation on shape- and composition-dependent properties but also for potential applications in photocatalysis, electrocatalysis, photonics, etc.
\end{abstract}

\title{
Keywords
}

concave AgI nanoparticles, photocatalysis, controlled etching, silver halide 


\section{Introduction}

Photocatalysis have attracted intensive interest in the past few decades due to their importance in supporting renewable energy and cleaning environmental pollutions [1-5]. Performances of the photocatalsyts are usually determined by a number of physical parameters, among which size and shape are critical ones to be optimized to maximize their performance in applications [6-10]. In some cases, shape control allows one to tailor the properties with a greater versatility than can be achieved otherwise. For example, the reactivity and selectivity of a nanoparticular catalyst can be significantly influenced by controlling its shape that determines the crystallographic facets exposed on the surfaces of the nanocrystal and the number of atoms located at edges or corners $[11,12]$. Compared to the enormous success for shape-controlled synthesis of micro/nanocrystals with convex surfaces, synthesis of nanocrystals with a concave surface is still in an infancy stage of development. A concave structure suggests curving in or hollowed inward from the surface and thus presence of regions with negative curvature. Owing to the potential high-index facets and negative curvature, nanocrystals with concave surfaces are expected to show unexplored or substantially enhanced performance relative to their convex counterparts. However, due to the more complicated structures of compound crystals, until now, the reports on the syntheses of concave micro/nanocrystals are primarily focused on noble metals [13]. There only have been few reports on concave compound crystals. For instance, $\mathrm{Ye}$ and coworkers synthesized concave trisoctahedral $\mathrm{Ag}_{3} \mathrm{PO}_{4}$ microcrystals based on the heteroepitaxial growth procedure, and the as-made sample exhibited better photocatalytic performance towards degradation of organic contaminants than the corresponding cubic $\mathrm{Ag}_{3} \mathrm{PO}_{4}$ microcrystals [14]. Huang et al. synthesized $\mathrm{AgCl}$ concave cubes by preferential overgrowth of $\mathrm{AgCl}$ cubes along the $\langle 111\rangle$ and $\langle 110\rangle$ directions with a wet chemical oxidation method, which showed improved photocatalyitc activity in $\mathrm{O}_{2}$ evolution than the original cubes [15]. Nevertheless, the controlled synthesis of concave compound micro/nanocrystals with improved performance still remains a challenge.

Silver iodide (AgI) with a bandgap of $2.80 \mathrm{eV}$ and a rich phase diagram [16] has been received much attention due primarily to its classical superior fast ionic conductivity $[17,18]$ and promising applications in visible-light-responsive photocatalysis [19]. AgI nanocrystals with several well-defined shapes including rod, tetrahedron and plate have been synthesized in colloidal solutions [20-23]. The syntheses are usually time-consuming, for example, several days are required to 
produce AgI nanoplates. Herein, we report a one-pot route for the synthesis of concave AgI sub-microcrystals through simple etching of spherical AgI particles in a solution containing ethylenediamine (EDA), absolute alcohol and polyvinylpyrrolidone (PVP) at a mild temperature. The morphology and size of AgI vary according to the amount of $\mathrm{I}^{-}$ions added to the reaction solution. It was found that the addition rate of $\mathrm{I}^{-}$ions, EDA and PVP play an important role in determining the quality of the obtained products. Furthermore, the concave AgI sub-microparticles exhibited significantly enhanced photocatalytic activity in comparison with the spherical ones.

\section{Experimental}

\subsection{Materials}

Silver acetate $\left(\mathrm{CH}_{3} \mathrm{COOAg}\right)$, potassium iodide (KI), ethylenediamine (EDA), absolute ethanol, and polyvinylpyrrolidone (PVP, MW = 40000) were purchased from Sinopharm Chemical Reagent Co., Ltd (Shanghai, China). All the reagents were used as received without further purification.

\subsection{Synthesis of concave and spherical AgI microcrystals}

The AgI concaves were synthesized by using a controlled precipitation reaction in a mixed solvent of EDA and absolute ethanol. In a typical synthesis, $9 \mathrm{~mL}$ of absolute ethanol, $1 \mathrm{~mL}$ of EDA, $50 \mathrm{mg}$ of PVP and $60 \mathrm{mg}$ of $\mathrm{CH}_{3} \mathrm{COOAg}(0.36 \mathrm{mmol})$ were placed in a $100-\mathrm{mL}$ flask sequentially, and the solution was kept at $30{ }^{\circ} \mathrm{C}$ under magnetic stirring. After PVP and $\mathrm{CH}_{3} \mathrm{COOAg}$ were completely dissolved, $5 \mathrm{~mL}$ ethanol solution of $\mathrm{KI}(0.084 \mathrm{mmol} / \mathrm{mL})$ was introduced to the above solution at a rate of $1 \mathrm{~mL} / \mathrm{min}$ with a syringe pump. After the addition was accomplished, the mixture was incubated for additional $10 \mathrm{~min}$. The as-synthesized product was collected by centrifugation, and rinsed 6 times with distilled water. The progress for the preparation of spherical AgI was similar except that $4.2 \mathrm{~mL}$ ethanol solution of KI with the same concentration as above was introduced into the reaction system.

\subsection{Characterization}

The crystalline structures of the synthesized particles were examined with X-ray powder diffraction (XRD) on a Philips X'Pert diffractometer with $\mathrm{Cu} K \alpha$ radiation $(\lambda=0.15418 \mathrm{~nm})$. Morphologies of the particles were studied on a Hitachi S-4800 field emission scanning electronic microscopy (FESEM). X-ray photoelectron spectroscopy (XPS) was performed with a Perkin-Elmer PHI-5300 spectrometer using non monochromatized $\mathrm{Mg} \mathrm{K} \alpha$ radiation as excitation source in a vacuum of $10^{-7} \mathrm{~Pa}$. Optical absorption spectra were recorded on a UV-vis spectrometer (UV-2600, 
Shimadzu, Japan) over a spectral range of $200-1000 \mathrm{~nm}$.

\subsection{Evaluation of photocatalytic performance}

The photocatalytic performance of the as-synthesized AgI nanocomposites was evaluated by the degradation of $\mathrm{RhB}$ dye under visible light irradiation $(\lambda \geq 400 \mathrm{~nm})$ using a $300 \mathrm{~W}$ Xe arc lamp (PLS-SEX300/300UV) with a UV-cutoff filter. The distance between the lamp and sample was 25 $\mathrm{cm}$. In a typical reaction, the as-synthesized $\mathrm{AgI}$ particles $(\sim 80 \mathrm{mg})$ were dispersed in $30 \mathrm{~mL}$ of an aqueous solution of $\mathrm{RhB}(20 \mathrm{mg} / \mathrm{L})$. The resulting dispersion was kept in the dark for $1 \mathrm{~h}$ with magnetic stirring to reach the adsorption-desorption equilibrium at room temperature. The degradation process of RhB was monitored by UV-vis spectra. After the reaction was completed, the catalyst was collected by centrifuging the suspension to be reused to catalyze a new reaction. For comparison, the investigations on both concave and spherical AgI particles were carried out.

\subsection{Photoelectrochemical measurements}

Photoelectrical response and electrochemical impedance spectroscopy (EIS) measurements were carried out using typical three-electrode cell consisting of a working electrode, a Pt gauze counter electrode, and a saturated calomel reference electrode (SCE) performed using an electrochemical workstation (CHI 660E, CH Instruments). The working electrodes were prepared as an indium-tin oxide glass (ITO) decorated with catalyst samples were used as the working electrode. In a typical procedure, $10 \mathrm{mg}$ of $\mathrm{AgI}$ catalyst was firstly dispersed in $0.5 \mathrm{~mL}$ of water containing polyvinylidene Fluoride (PVDF) solution $(50 \mu \mathrm{L}, 5 \mathrm{wt} \%)$, and the mixture was ultrasonicated to produce a homogeneous ink. $20 \mu \mathrm{L}$ of the catalyst ink (containing $0.4 \mathrm{mg}$ of catalyst) was spreaded onto an ITO glass with an active area of $c a .1 \mathrm{~cm}^{2}$ (loading density: $0.4 \mathrm{mg} / \mathrm{cm}^{2}$ ). To ensure the attachment of catalyst onto the ITO glass, the photo-electrodes were cured at $100{ }^{\circ} \mathrm{C}$ for $3 \mathrm{~h}$. The photocurrents were measured using an electrochemical analyzer with a standard three-electrode system using the prepared samples as the working electrodes, a Pt foil as the counter electode, and a saturated calomel reference electrode $(\mathrm{SCE})$. The visible light $(\lambda \geq 400 \mathrm{~nm})$ was produced by a $300 \mathrm{~W}$ Xe arc lamp (PLS-SEX300/300UV) with a UV-cutoff filter, and $0.5 \mathrm{M} \mathrm{NaSO}_{4}$ aqueous solution was used as the electrolyte. The electrochemical impedance spectroscopy (EIS) measurements were carried out in the same system and recorded over a frequency range of $10^{5}-0.01 \mathrm{~Hz}$ with ac amplitude of $5 \mathrm{mV}$ at open circuit potential $(0.213 \mathrm{~V})$ whereas the electrolyte was consisting of $\quad 0.5 \mathrm{M} \mathrm{NaSO}_{4}$ aqueous solution with $0.6 \mathrm{mM} \mathrm{K}_{3}\left[\mathrm{Fe}(\mathrm{CN})_{6}\right] / \mathrm{K}_{4}\left[\mathrm{Fe}(\mathrm{CN})_{6}\right](1: 1)$ as redox probe. The cyclic voltammograms $(\mathrm{CV})$ were 
also measured with a scanning rate of $10 \mathrm{mV} / \mathrm{s}$.

\section{Results and discussion}

Figure 1 presents the typical stages involved in the formation of concave AgI sub-microparticles derived from the AgI spherical particles. The synthesis starts with a precipitation reaction between $\mathrm{I}^{-}$ and $\mathrm{Ag}^{+}$ions in the presence of PVP as a capping agent and ethylenediamine (EDA) as a releasing-controlled agent of $\mathrm{Ag}^{+}$. With the addition of $\mathrm{I}^{-}$ions to the reaction solution containing $\mathrm{Ag}^{+}$ ions, spherical AgI nanoparticles are formed rapidly, and they continuously grow larger until the ratio of $\mathrm{I}^{-} / \mathrm{Ag}^{+}$reaches 1 . When the amount of $\mathrm{I}^{-}$ions in the reaction solution surpasses the stoichiometry necessity, i.e. $\mathrm{I}^{-} / \mathrm{Ag}^{+}>1$, the excessive $\mathrm{I}^{-}$ions begin to etch the surfaces of the sub-microspheres by following the reaction $\mathrm{AgI}+\mathrm{I}^{-} \rightarrow \mathrm{AgI} 2^{-}$. This chemical etching results in the formation of concave surfaces in AgI particles. The experimental details are described in the experimental section.

Figure $2 \mathrm{~A}$ and $\mathrm{B}$ are the SEM images of the products formed at the $\mathrm{I}^{-} / \mathrm{Ag}^{+}$of 1 , clearly showing their spherical morphology with rough surfaces and relative uniform size with an average diameter of $498 \mathrm{~nm}$ (Supporting Information, Figure S1B). The products obtained from $\mathrm{I}^{-} / \mathrm{Ag}^{+}=1.16$ exhibit concave surfaces in the AgI particles (Figure 2C, D). The dependence of morphology of the AgI particles on the $\mathrm{I}^{-} / \mathrm{Ag}^{+}$indicates that the feeding amount of $\mathrm{I}^{-}$ions plays an essential role in determining the shape of the products. The concave AgI particles have sizes (an average of $373 \mathrm{~nm}$ ) much smaller than the spheres, indicating that the concaving process is indeed caused by partially dissolving AgI spherical particles. For comparison, the samples synthesized without PVP or EDA display irregular shapes (Supporting Information, Figure S2), showing that the existence of both PVP and EDA in the reaction solution is critical for producing high quality AgI particles with either spherical or concave morphology. The elemental composition and chemical state for the AgI particles shown in Figure 2C were analyzed by XPS (Supporting Information, Figure S3). The survey spectrum indicates that the obtained samples are mainly composed of $\mathrm{Ag}$ and I. The oxygen signal may originate from the adsorbed $\mathrm{O}_{2}$ or $\mathrm{H}_{2} \mathrm{O}$ on the sample surfaces. The element $\mathrm{Ag}$ is present in the form of $\mathrm{Ag}(\mathrm{I})$, which corresponds to the binding energy of 373.9 and $367.9 \mathrm{eV}$ for $\mathrm{Ag} 3 \mathrm{~d} 3 / 2$ and Ag 3d5/2 in AgI, respectively (Supporting Information, Figure S3B). The I 3d3/2 and I 3d5/2 levels appear at the binding energy of 630.6 and $619.1 \mathrm{eV}$, respectively (Supporting Information, Figure S3C), which well match previous report for Ag:I with a stoichiometric ratio [24]. 
The morphological evolution was tracked by analyzing the products formed at different reaction times (corresponding to different feeding amount of $\mathrm{I}^{-}$ions) with SEM imaging. From the SEM images shown in Figure 3, we can notice that the well-defined spheres with a mean diameter of 308 $\mathrm{nm}$ were formed at short time of $1 \mathrm{~min}$, at which the amount of $\mathrm{I}^{-}$ions added to the reaction solution was much less than that of $\mathrm{Ag}^{+}$. As more $\mathrm{I}^{-}$ions were added to the reaction solution, the $\mathrm{AgI}$ spheres grow to form larger ones, such as the particles with diameters of $\sim 510 \mathrm{~nm}$ formed at 3 min (Figure 3B). The spheres continuously grow larger until the overall amount of $\mathrm{I}^{-}$equals to that of $\mathrm{Ag}^{+}$. $\mathrm{At}^{5}$ min when the amount of $\mathrm{I}^{-}$is excessive over $\mathrm{Ag}^{+}, \mathrm{AgI}$ spherical particles can be partially dissolved, resulting in the formation of concave shape (Figure 3C). The size $(\sim 400 \mathrm{~nm})$ of the concave particles becomes smaller than the spheres (the dependence of particles size on time is shown in Supporting Information, Figure S4). Incubating the concave AgI particles in the original reaction solution for additional 10 min can smoothen their surfaces and lead the particles to be uniform in size (Figure 2D). However, if too much quantity of $\mathrm{I}^{-}$ions was added (i.e., $8 \mathrm{~mL}$ of $\mathrm{KI}$ solution, $\mathrm{I}^{-} / \mathrm{Ag}^{+}=1.84$ ), irregular AgI particles were resulted due to fragmentation (Supporting Information, Figure S5). Therefore, carefully controlling the ratio of $\mathrm{I}^{-} / \mathrm{Ag}^{+}$is very important to synthesize uniform concave AgI particles.

The sharp diffraction peaks in the XRD patterns (Figure 4) show that both concave and spherical samples are well crystallized. All the diffraction peaks can be indexed to the hexagonal phase of $\beta$-AgI with lattice parameters of $\mathrm{a}=\mathrm{b}=4.59 \AA, \mathrm{c}=7.50 \AA$ [JCPDS card No. 83-0582]. There is no obvious difference between the spherical and concave-shaped particles. By correcting the peak width with instrumental parameter, the crystallite sizes of the concave and spherical particles are estimated to be $357 \mathrm{~nm}$ (concave) and $444 \mathrm{~nm}$ (sphere) according to the Scherrer equation, respectively. These values are close to the observed sizes in SEM images, indicating that each particle is a single crystal regardless of their morphology. As a result, surface area of individual particles is mainly determined by their morphology. It is expected that formation of concaved surfaces in a spherical particle can increase the overall surface area. Brunauer-Emmett-Teller (BET) gas adsorption/desorption measurements clearly show that surface area of the spherical particles of Figure $2 \mathrm{~B}$ increases from $1.68 \mathrm{~m}^{2} / \mathrm{g}$ to $2.40 \mathrm{~m}^{2} / \mathrm{g}$ when they are transformed into concaved particles of Figure 2D (Supporting Information, Figure S6). 
Optical absorption spectra (Supporting Information, Figure S7) of the two samples indicate that the concave AgI particles exhibit stronger absorption with an absorption peak around $490 \mathrm{~nm}$ than the spherical AgI particles with an absorption band position around $586 \mathrm{~nm}$. This spectral difference might be ascribed to their difference in morphology. The absorbed photons in AgI particles can efficiently induce charge separation to generate highly active energetic charges that can transfer to species adjacent to the AgI particles. For example, electron spin resonance (ESR) spectroscopy of an aqueous solution of 5,5-dimethyl-1-pyrroline-N-oxide (DMPO) in the presence of concave AgI particles clearly shows the effective formation of nitroxide radicals by reacting with the photogenerated electrons under photo-illumination of visible light (Supporting Information, Figure S8). Such efficient charge generation and transfer in the synthesized AgI particles enables them to be a class of efficient solar-driven photocatalysts. The photocatalytic performance of the as-synthesized concave AgI particles was evaluated by the degradation of RhB dye under visible light. A series of absorption spectra of the $\mathrm{RhB}$ solution were collected before and after a mixture of as-prepared catalyst and $\mathrm{RhB}$ molecules had been illuminated under visible-light at different reaction times (Supporting Information, Figure S9A). The absorption of $\mathrm{RhB}$ decreases steadily as the reaction proceeds, and the color of RhB solution changes from pink to colorless. These features indicate RhB molecules can be rapidly degraded within 5 min under visible light irradiation with the assistance of the concave $\mathrm{AgI}$ particles. The function of $\ln \left(C / C_{0}\right)$ versus reaction time $(t)$ displays a linear relationship (Figure 5B and Figure S9B), indicating that the reaction follows the first-order kinetics with a rate constant determined to be $0.79 \mathrm{~min}^{-1}$. In contrast, the experiment under the same conditions with spherical AgI particles was also carried out, showing that only $17.9 \%$ of $\mathrm{RhB}$ molecules are decomposed for $5 \mathrm{~min}$. As well known, the adsorption capacity for the dye molecules is one of the crucial factors for catalytic activity [25-28]. The adsorption abilities of the same amount of the as-prepared concave AgI and spherical AgI particles are shown in Figure 5A: the concave AgI particles exhibits a much higher adsorption capacity than the spherical AgI particles (i.e., 19.1\% versus $12.1 \%$ ). The improved adsorption capacity is ascribed to the larger surface area of concaved particles and is beneficial for the enhancement of photocatalytic activity. Another reason for enhanced activity is that the concave particles have stronger optical absorption coefficient than the spherical ones in the visible region, which endows the concave particles to produce more photo-generated carriers. 
For practical applications, stability is also important for photocatalysts. To test the stability of the concave AgI particles, the particles were collected by centrifuging the reaction solution and reused to catalyze the next cycles after one reaction was accomplished. The degradation kinetics of $\mathrm{RhB}$ for 5 successive runs of reactions using the same batch of concave AgI particles were similar (Supporting Information, Figure S10), indicating that the photocatalytic efficiency of the as-synthesized concave AgI particles maintained well after 5 times. The slightly decreased activity is due to the inevitable loss of catalyst over the recovering process.

In the process of oxidative degradation of $\mathrm{RhB}$, photo-generated positive charges (i.e., $h^{+}$) and other possible radical species (e.g., $\mathrm{O}_{2}{ }^{-}$, and $\cdot \mathrm{OH}$ ) formed from capturing energetic chargers by dissolved $\mathrm{O}_{2}$ and $\mathrm{H}_{2} \mathrm{O}$ molecules. In order to further elucidate the involved species in the degradation reaction, $p$-benzoquinone (PBQ $5 \mathrm{mM}$, scavenger for $\mathrm{O}_{2}{ }^{-}$), tert-butanol (TBA, $0.1 \mathrm{M}$, scavenger for $\cdot \mathrm{OH})$ and $\mathrm{NaHCO}_{3}\left(10 \mathrm{mM}\right.$, scavenger for $h^{+}$and adsorbed $\left.\cdot \mathrm{OH}\right)$ were selectively introduced to the reaction system. The addition of three scavengers into the system results in the strong depression of the degradation rate of $\mathrm{RhB}$ to some extent (Supporting Information, Figure S11), indicating that the $\mathrm{O}_{2} \cdot{ }^{-}, h^{+}$, adsorbed $\cdot \mathrm{OH}$ and free $\cdot \mathrm{OH}$ in the solution are more likely the active species. The addition of only $\mathrm{NaHCO}_{3}$ has no significant influence on the activity of the catalyst, indicating that holes $\left(h^{+}\right)$ play a minor role in the present reaction. When PBQ and TBA were added, the degradation was dramatically depressed, confirming that the $\mathrm{O}_{2}{ }^{-}$and $\cdot \mathrm{OH}$ are the major oxidation species [19].

To better understand the enhanced photocatalytic activity for both concave and spherical AgI particles, transient photocurrent responses of the electrodes made of the corresponding AgI particles were recorded for several on/off cycles of intermittent irradiation of visible light $(\lambda \geq 400 \mathrm{~nm})$ at a bias potential of $0.15 \mathrm{~V}$. Figure $6 \mathrm{~A}$ shows a comparison of the photocurrent-time $(I-t)$ curves of different electrodes. The photocurrent response of concave AgI is prompt, steady, and reproducible, and the short circuit photocurrent density is 3 times higher than that of spherical counterpart, indicating that concave particles is much more sensitive to visible light and generated more photo-carriers [29]. Electrochemical impedance spectra (EIS) is also a powerful technique to study the charge transfer process involved in the three-electrode system. The high-frequency response is due to the charge transfer (or electrochemical reaction) at the Pt counter electrode, while the intermediate-frequency response is associated with the electron transport and transfer at the catalyst electrode interface [30-32]. As shown in Figure 6B, the typical EIS were presented as Nyquist plots 
and it is observed that concave AgI has a smaller semicircle than spherical one in the middle-frequency region, which further proves the faster interfacial electron transfer over the concave catalyst [33]. Meanwhile, The concave AgI presents a larger current density than spherical $\mathrm{AgI}$ following the analyses of cyclic voltammetric (CV) curves (Figure 5C). In fact, the current density for concave AgI exhibits an enhancement of 2.2-folds compared to that of spherical AgI sample, further demonstrating the concave AgI possesses significantly enhanced electron transfer rate [34]. The enhancement can be ascribed to the unique characteristics of concave particles such as their surface curvature. These figures of merits are consistent with the improved photocatalytic performance of the concave AgI particles in comparison with the spherical particles.

\section{Conclusion}

In summary, we have demonstrated a facile strategy for the synthesis of concave AgI particles by a controllable etching of AgI spherical particles with excessive $\mathrm{I}^{-}$ions. Compared to spherical particles, the concave AgI sub-microparticles exhibit a much higher efficiency in photocatalyzing degradation of $\mathrm{RhB}$. The concave materials are expected to be useful not only for the fundamental investigation on shape- and composition-dependent behaviors but also for many promising applications such as photocatalysis, electrocatalysis, photonics, superionic conductor and nanoelectronics.

\section{Acknowledgements}

The authors gratefully acknowledge the financial support by National Natural Science Foundation of China (Grant No. 21001116), China Postdoctoral Science Foundation (2013M541963), and the Fundamental Research Funds for the Central Universities. Use of the Center for Nanoscale Materials was supported by the U. S. Department of Energy, Office of Science, Office of Basic Energy Sciences, under Contract No. DE-AC02-06CH11357.

\section{References}

[1] J. Tang, Z. Zou, J. Ye, Angew. Chem. Int. Ed. 43 (2004) pp. 4463-4466.

[2] T. P. Yoon, M. A. Ischay, J. Du, Nat. chem. 2 (2010) pp. 527-532.

[3] X. Chen, S. Shen, L. Guo, S. S. Mao, Chem. Rev. 110 (2010) pp. 6503-6570.

[4] S. N. Habisreutinger, L. Schmidt Mende, J. K. Stolarczyk, Angew. Chem. Int. Ed. 52 (2013) pp. 7372-7408. 
[5] H. Tong, S. Ouyang, Y. Bi, N. Umezawa, M. Oshikiri, J. Ye, Adv. Mater. 24 (2012) pp. 229-251.

[6] H. Kisch, Angew. Chem. Int. Ed. 52 (2013) pp. 812-847.

[7] A. Testino, I. R. Bellobono, V. Buscaglia, C. Canevali, M. D'Arienzo, S. Polizzi, R. Scotti, F. Morazzoni, J. Am. Chem. Soc. 129 (2007) pp. 3564-3575.

[8] B. Wiley, Y. G. Sun, Y. N. Xia, Acc. Chem. Res. 40 (2007) pp. 1067-1076.

[9] T. K. Sau, A. L. Rogach, Adv. Mater. 22 (2010) pp. 1781-1804.

[10] S. Linic, P. Christopher, D. B. Ingram, Nat. Mater. 10 (2011) pp. 911-921.

[11] R. Narayanan, M. A. El-Sayed, Nano Lett. 4 (2004) pp. 1343-1348.

[12] A. Kolmakov, D. O. Klenov, Y. Lilach, S. Stemmer, M. Moskovits, Nano Lett. 5 (2005) pp. 667-673.

[13] H. Zhang, M. S. Jin, Y. N. Xia, Angew. Chem. Int. Ed. 51 (2012) pp. 7656-7673.

[14] Z. B. Jiao, Y. Zhang, H. C. Yu, G. X. Lu, J. H. Ye, Y. P. Bi, Chem. Commun. 49 (2013) pp. 636-638.

[15] Z. Z. Lou, B. B. Huang, X. Y. Qin, X. Y. Zhang, H. F. Cheng, Y. Y. Liu, S. Y. Wang, J. P. Wang, Y. Dai, Chem. Commun. 48 (2012) pp. 3488-3490.

[16] H. S. Liu, Y. H. Wang, C. C. Li, C. Y. Tai, Chem. Eng. J. 183 (2012) pp. 466-472.

[17] R. Makiura, T. Yonemura, T. Yamada, M. Yamauchi, R. Ikeda, H. Kitagawa, K. Kato, M. Takata, Nat. Mater. 8 (2009) pp. 476-480.

[18] R. J. Cava, E. A. Rietman, Phys. Rev. B 30 (1984) pp. 6896-6902.

[19] C. Hu, T. Peng, X. Hu, Y. Nie, X. Zhou, J. Qu, H. He, J. Am. Chem. Soc. 132 (2010) pp. 857-862.

[20] Y. G. Guo, J. S. Lee, J. Maier, Adv. Mater. 17 (2005) pp. 2815-2819.

[21] C. H. B. Ng, W. Y. Fan, J. Phys. Chem. C 111 (2007) pp. 2953-2958.

[22] S. Feng, H. Xu, L. Liu, Y. Song, H. Li, Y. Xu, J. Xia, S. Yin, J. Yan, Colloids Surf., A 410 (2012) pp. 23-30.

[23] W. Jiang, C. An, J. Liu, S. Wang, L. Zhao, W. Guo, J. Liu, Dalton Trans. 43 (2013) pp. 300-305.

[24] Z. Zheng, A. R. Liu, S. M. Wang, B. J. Huang, X. M. Ma, H. X. Zhao, D. P. Li, L. Z. Zhang, Mater. Res. Bull. 43 (2008) pp. 2491-2496. 
[25] H. Zhang, X. Lv, Y. Li, Y. Wang, J. Li, ACS Nano 4 (2010) pp. 380-386.

[26] M. Zhu, P. Chen, M. Liu, ACS Nano 5 (2011) pp. 4529-4536.

[27] L. W. Zhang, H. B. Fu, Y. F. Zhu, Adv. Funct. Mater. 18 (2008) pp. 2180-2189.

[28] W. Morales, M. Cason, O. Aina, N. R. de Tacconi, K. Rajeshwar, J. Am. Chem. Soc. 130 (2008) pp. 6318-6319.

[29] S. Zhuo, Y. Xu, W. Zhao, J. Zhang, B. Zhang, Angew. Chem. Int. Ed. 125 (2013) pp. 8764-8768.

[30] B.-L. He, B. Dong, H.-L. Li, Electrochem. Commun. 9 (2007) pp. 425-430.

[31] A. Jana, C. Bhattacharya, J. Datta, Electrochim. Acta 55 (2010) pp. 6553-6562.

[32] J. Yu, J. Fan, B. Cheng, J. Power Sources 196 (2011) pp. 7891-7898.

[33] H. Zhang, X. Lv, Y. Li, Y. Wang, J. Li, ACS Nano 4 (2010) pp. 380-386.

[34] X. Pan, Y. Zhao, S. Liu, C. L. Korzeniewski, S. Wang, Z. Fan, ACS Appl. Mater. Inter. 4 (2012) pp. 3944-3950. 


\section{Figure Captions}

Figure 1. A schematic illustration for the growth of the AgI spheres and their transformation to the AgI concave particles through the $\mathrm{I}^{-}$ions etching effect.

Figure 2. (A, B) Low and high magnification of SEM images of the as-synthesized AgI spheres at the stoichiometric ratio of $\mathrm{I}^{-}$to $\mathrm{Ag}^{+}$; (C, D) Low and high magnification of SEM images of the as-synthesized $\mathrm{AgI}$ concaves at ratio of $\mathrm{I}^{-} / \mathrm{Ag}^{+}=1.16$, which was obtained with the incubation of the product for additional $10 \mathrm{~min}$ after the addition of $\mathrm{I}^{-}$ions was completed.

Figure 3. SEM images of the as-prepared AgI particles at different reaction times: (A) 1 min (I- / $\left.\mathrm{Ag}^{+}=0.23\right)$, (B) $3 \mathrm{~min}\left(\mathrm{I}^{-} / \mathrm{Ag}^{+}=0.69\right),(\mathrm{C}) 5 \mathrm{~min}\left(\mathrm{I}^{-} / \mathrm{Ag}^{+}=1.16\right)$. The scale bars in the insets are all $300 \mathrm{~nm}$.

Figure 4. XRD patterns of the concave (blue curves) and spherical (black curves) AgI particles.

Figure 5. The comparisons of the adsorption capacity (A) and degradation of RhB solution (B) over concave and spherical AgI particles. The result clearly shows that the concave particles exhibit superior performance to the spherical counterparts.

Figure 6. (A) Transient photocurrent responses, (B) electrochemical impedance spectroscopy, and (C) cyclic voltammograms of the concave (blue curves) and and spherical (black curves) AgI particles. The current densities in (A) and (C) were normalized against the apparent electrode areas. 
Vitae

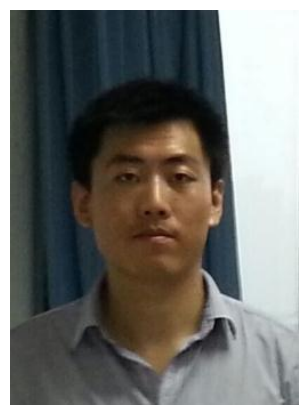

Junxue Liu is currently a Ph.D. candidate at China University of Petroleum (UPC), He received his M.S. degree in chemistry in 2014 (with Prof. Changhua An), from the China University of Petroleum (UPC). His currently scientific interests focus on controlled synthesis and catalytic applications of nanophotocatalysts.

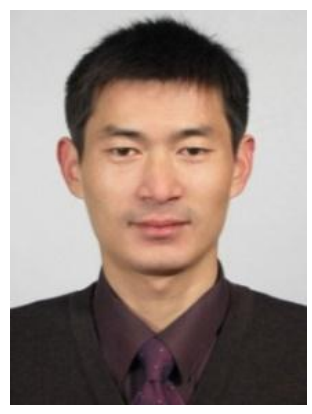

Changhua An received his $\mathrm{PhD}$ degree from University of Science and Technology of China (USTC) in 2003, then he worked as postdoctoral research fellow at Seoul National University, Korea from 2004 to 2005 . He has been an associate professor at China University of Petroleum (UPC) from 2005 . $\mathrm{He}$ worked as a visiting scholar at University of Illinois at Urbana-Champaign, USA from 2009 to 2010. In 2013, he was promoted to Full Professor of Materials Science. His research interests focus on the synthesis, characterization, modifications, and applications of nanophotocatalysts for solar-chemical energy transformation.

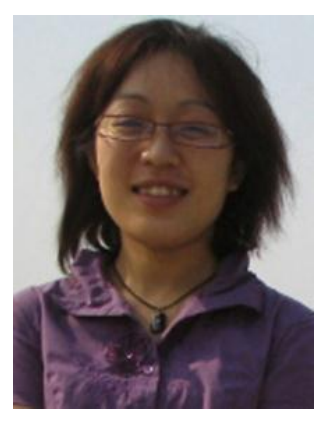

Shutao Wang received her $\mathrm{PhD}$ in Chemistry from University of Science and Technology of China (USTC) in 2006. Then she joined the faculty at China University of Petroleum (UPC), where she is currently an associate professor. Her research involves the functional inorganic nanocrystals, with emphasis on energy-related photocatalysts and hydrogenation nanocatalysts.

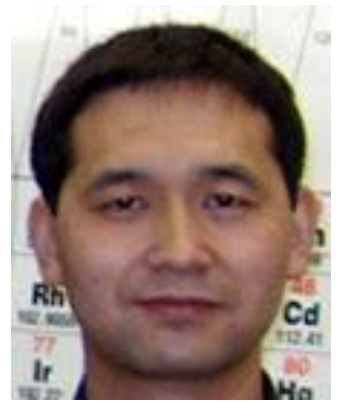

Jun Zhang received his B.Sc. degree in inorganic and M.Sc. degree in organic Chemistry from Lanzhou University in 1996 and 2003, respectively. After he received his a Ph.D. degree in Material Chemistry from the State University of New York at Binghamton, he worked in Center for Nanoscale Materials(CNM) at Argonne National Laboratory with a CNM distinguished postdoctoral fellowship from 2010 to 
2013. He is currently a professor in the Chemical Engineering Department at China University of Petroleum. His research interests are on the synthesis, characterization, and surface functionalization of nanomaterials for energy-related applications.

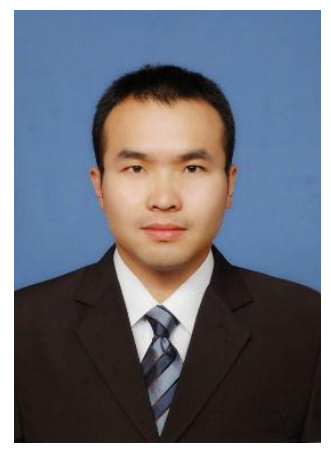

Zhaojie Wang received his $\mathrm{PhD}$ degree in Polymer Chemistry and Physics from Jilin University in 2013. He became a research assistant of China University of Petroleum in 2013 summer. His research interests focus on the one-dimensional functional nanomaterials.

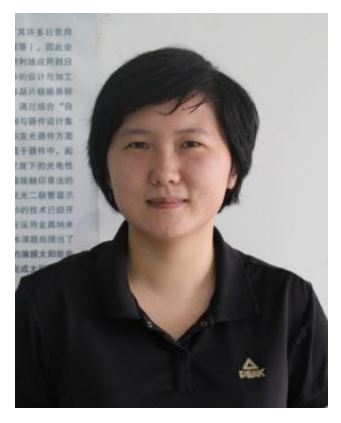

Ran Long was born in Anhui, China, in 1987. She received her B.S. in chemistry in 2009 and Ph.D. in inorganic chemistry in 2014 (with Prof. Yujie Xiong), both from the University of Science and Technology of China (USTC). She is currently a postdoctoral fellow working with Profs. Yujie Xiong and Li Song at the USTC. Her research interests focus on controlled synthesis and catalytic applications of metal nanocrystals.

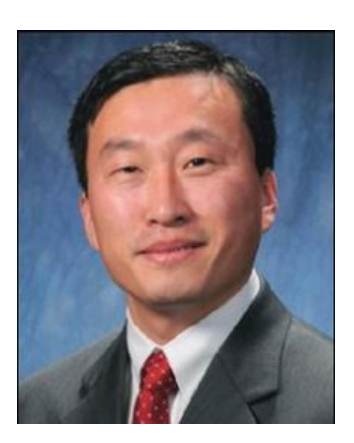

Yugang Sun received his $\mathrm{BS}$ and $\mathrm{PhD}$ degrees in chemistry from the University of Science and Technology of China (USTC) in 1996 and 2001, respectively. He is currently a staff scientist for the Center for Nanoscale Materials at Argonne National Laboratory. His current research interests focus on the synthesis of a wide range of nanostructures, including metal NPs with tailored properties, the development of in situ synchrotron X-ray techniques for real-time probing of nanoparticle growth, and the application of these nanomaterials in energy storage, photocatalysis, and sensing. 


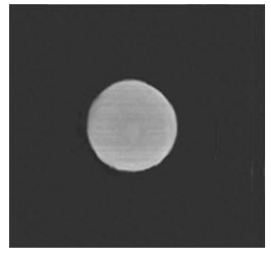

Agl sphere

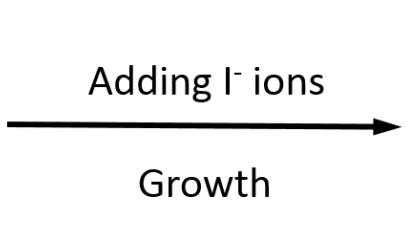

Agl sphere

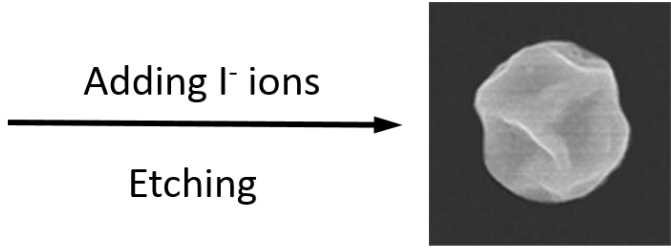

Agl concave

Figure 1
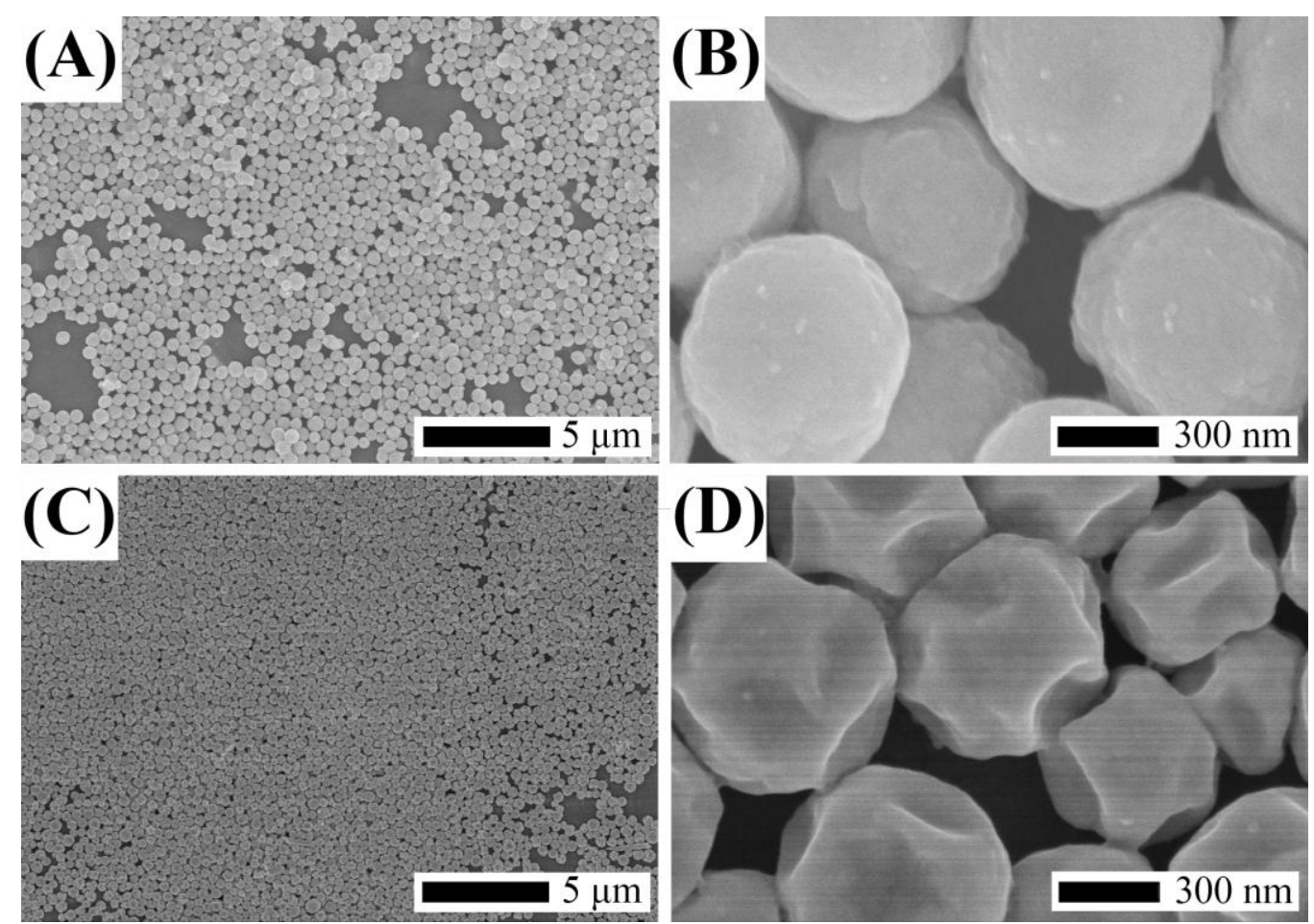

Figure 2 

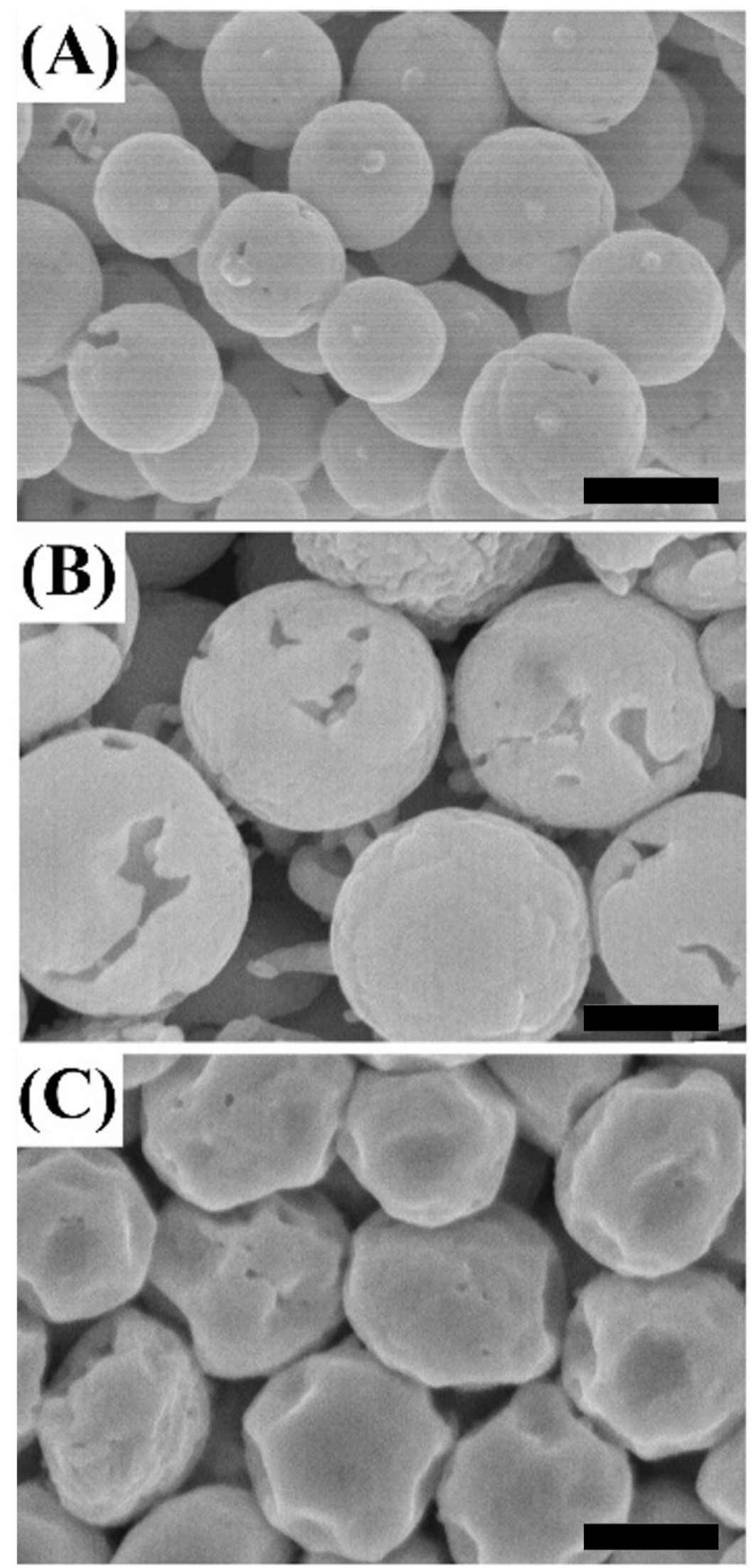

Figure 3 


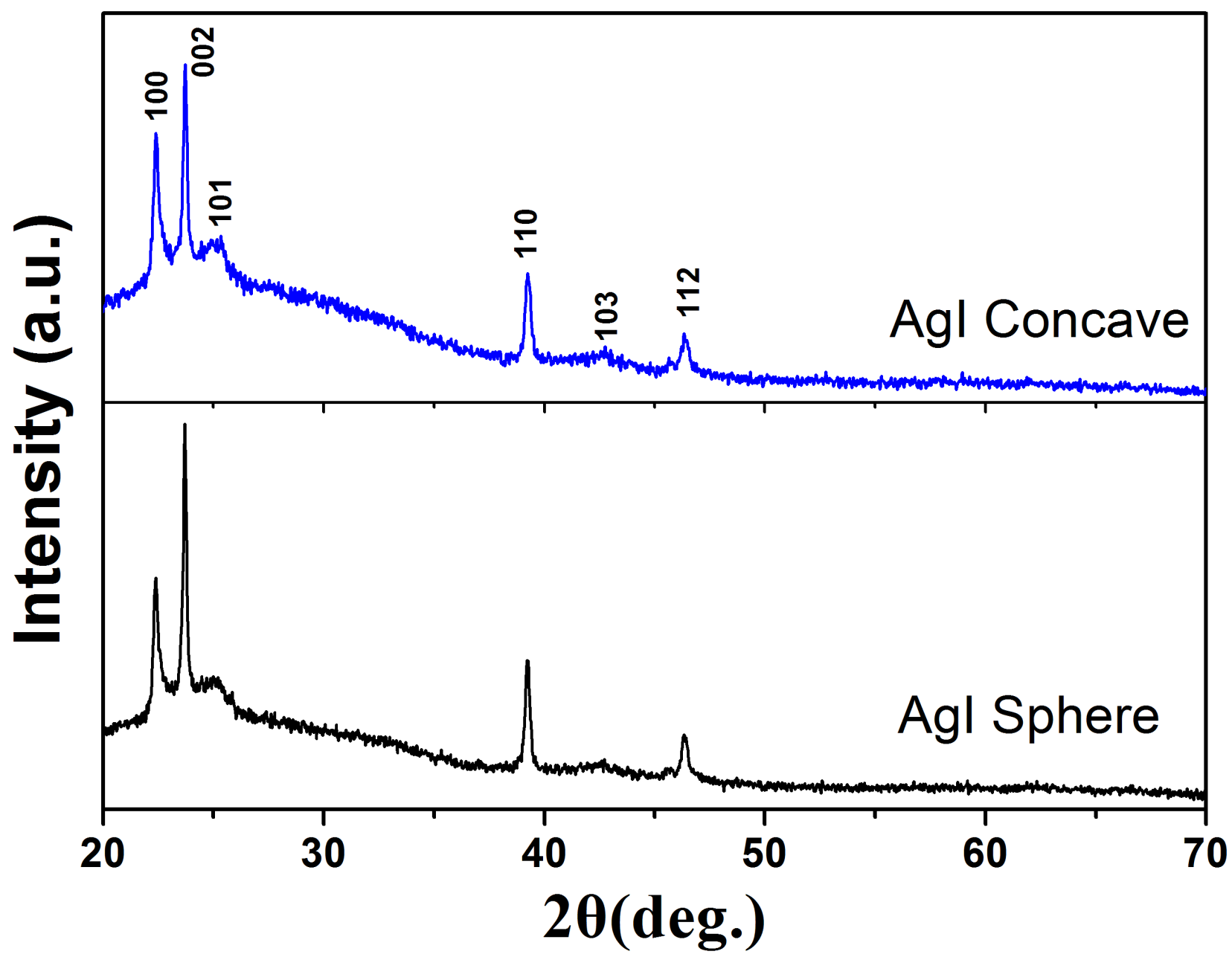

Figure 4 

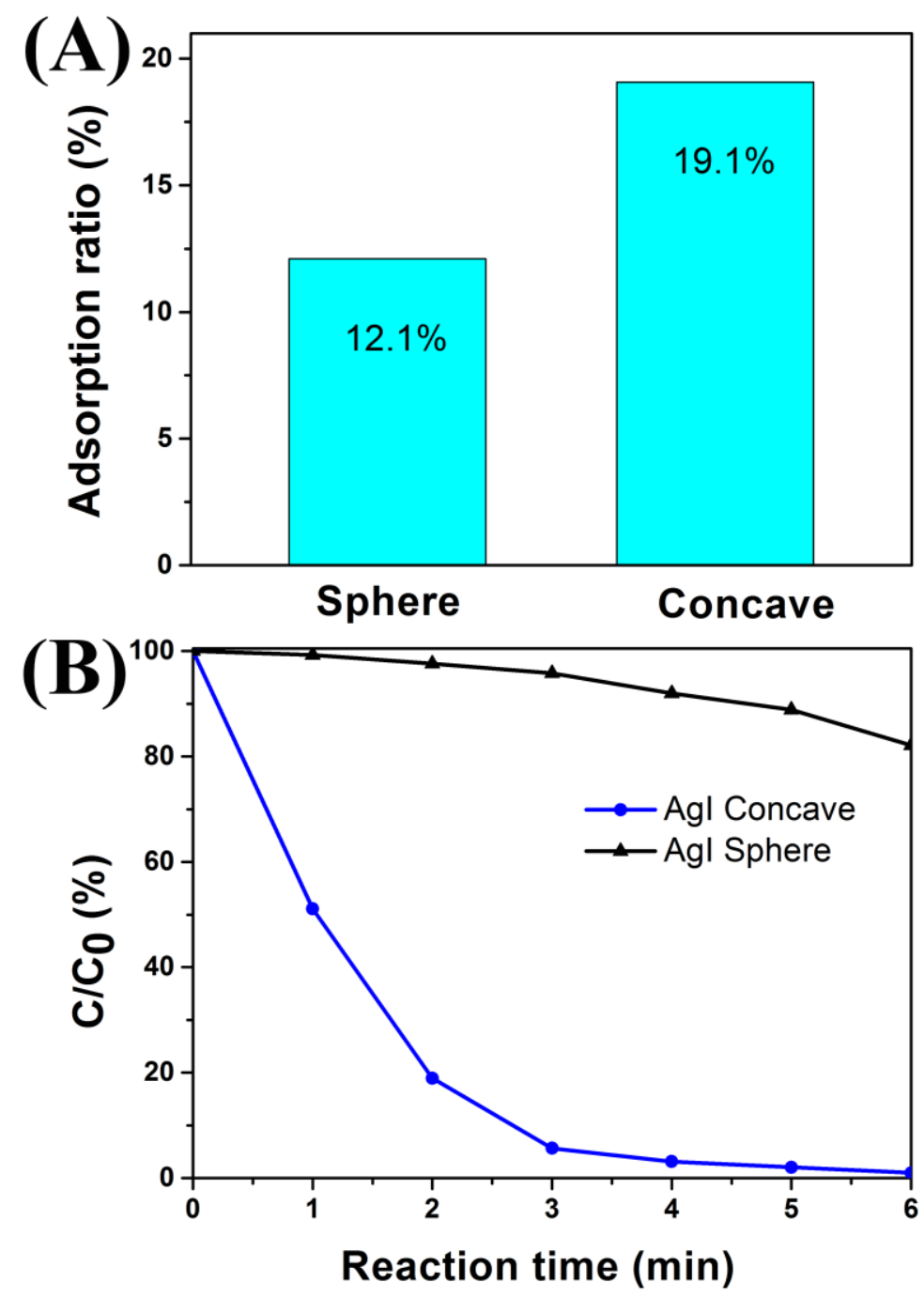

Figure 5 

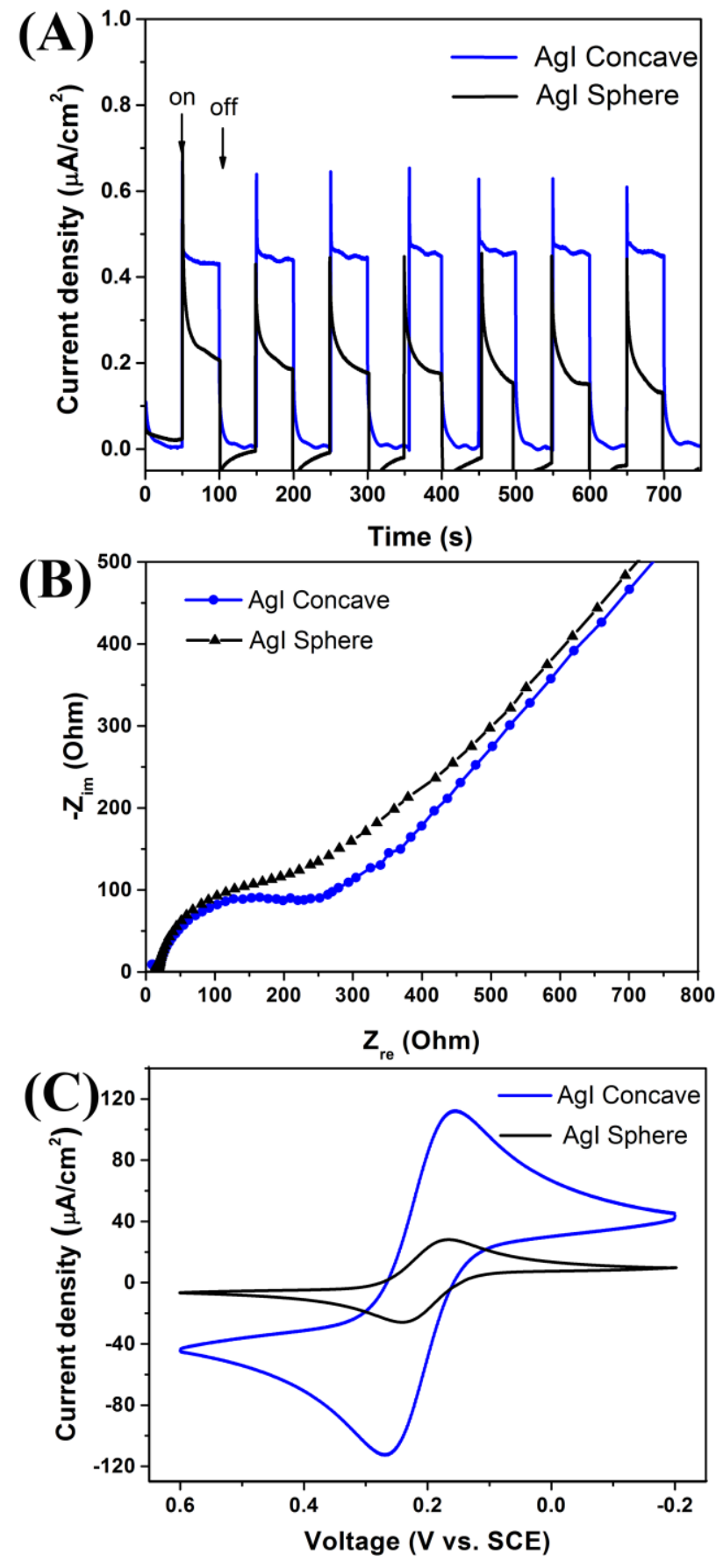

Figure 6 